\title{
No Evidence for Ceftobiprole-Induced Immune Hemolytic Anemia in Three Phase 3 Clinical Trials
}

This article was published in the following Dove Press journal:

Infection and Drug Resistance

\section{Kamal Hamed (D) \\ Tatiana Wiktorowicz \\ Maziar Assadi Gehr}

Basilea Pharmaceutica International Ltd., Basel, Switzerland
Correspondence: Kamal Hamed

Basilea Pharmaceutica International Ltd., Grenzacherstrasse 487, Basel 4005, Switzerland

$\mathrm{Tel}+4|6| 567 \mid 588$

Fax +4I 616061216

Email kamal.hamed@basilea.com
Purpose: Drug-induced immune hemolytic anemia (DIIHA) is a rare but serious adverse event associated with a number of drugs, including second- and third-generation cephalosporins. A positive direct antiglobulin test (DAT) is a reliable finding in DIIHA, but positive results without evidence of hemolysis can occur, particularly in hospitalized patients. There have been no reports of hemolytic anemia in four previous Phase 3 trials or from postmarketing surveillance of the advanced-generation, broad-spectrum cephalosporin, ceftobiprole. The aim of this analysis was to review the incidence of positive DAT results and any evidence of hemolytic anemia from three recent Phase 3 trials of ceftobiprole.

Patients and Methods: Patients were enrolled in three Phase 3 randomized controlled trials: 94 pediatric patients with pneumonia received ceftobiprole in the BPR-PIP-002 trial; 335 adults with acute bacterial skin and skin structure infections received ceftobiprole in the TARGET trial; and 201 adults with Staphylococcus aureus bacteremia have been randomized 1:1 to ceftobiprole or daptomycin \pm aztreonam in the ongoing ERADICATE trial. In all three trials, DAT results were obtained at baseline, and follow-up tests were performed either at the test of cure (TOC) visit (BPR-PIP-002), end-of-treatment (EOT) visit (TARGET), or both EOT and post-treatment Day 70 visits (ERADICATE).

Results: In the BPR-PIP-002 trial, five patients (all ceftobiprole treated) had a documented negative DAT result at baseline followed by a positive result at the TOC visit. One patient in the ongoing, blinded ERADICATE trial had a positive DAT result at both baseline and EOT. Results from other laboratory investigations showed no evidence of hemolytic anemia in these patients. No positive DAT results were reported in the TARGET trial.

Conclusion: No evidence of hemolytic anemia associated with ceftobiprole was observed in either adults or children across several indications in this analysis of three large Phase 3 trials.

Keywords: DIIHA, direct antiglobulin test, hemolysis, pneumonia, ABSSSIs, Staphylococcus aureus bacteremia

\section{Plain Language Summary}

Drug-induced immune hemolytic anemia is an extremely rare form of anemia. It occurs when red blood cells are destroyed by the immune system in response to treatment with certain pharmacological therapies. Evidence obtained over many decades has demonstrated that the second- and third-generation cephalosporin antibiotics are a leading cause of drug-induced hemolytic anemia. However, a similar association has not been reported for the advancedgeneration cephalosporins. In cases of drug-induced immune hemolytic anemia, a laboratory investigation known as the direct antiglobulin test (DAT; colloquially known as the direct Coombs' test) is usually positive. However, this test can be positive without evidence of hemolytic anemia, particularly in hospitalized patients, and results must be evaluated in the context of other laboratory or clinical findings. 
The objective of this analysis was to evaluate the frequency of positive DAT results and hemolytic anemia in patients treated with the advanced-generation cephalosporin, ceftobiprole. In three large Phase 3 trials in both adults and children, we found no evidence of hemolytic anemia in any patient treated with ceftobiprole. A small number of positive DAT results were reported at baseline and/or after treatment with ceftobiprole. However, in the absence of other laboratory or clinical findings indicative of hemolytic anemia, these results may simply reflect the fact that these patients had infections severe enough to warrant hospitalization. These results are consistent with earlier Phase 3 trials of ceftobiprole and other advancedgeneration cephalosporins. Our analysis provides further evidence that hemolytic anemia may not be associated with advancedgeneration cephalosporin treatment.

\section{Introduction}

Drug-induced immune hemolytic anemia (DIIHA) is a rare but serious complication of drug treatment, with an estimated incidence of approximately 1 case per million individuals, per year. ${ }^{1}$ The mechanism of DIIHA is complex and poorly understood, $^{2-4}$ and there is usually no relationship between drug dose and reaction. ${ }^{5}$ Most antibodies that cause DIIHA are categorized as drug-dependent, ie, they demonstrate reactivity only in the presence of drug. ${ }^{2}$ Less commonly, DIIHA is caused by drug-independent antibodies, ie, antibodies that are capable of in vitro reactivity in the absence of drug. ${ }^{2}$ Antibodies may be directed against the drug, a neoantigen formed by the drug and red blood cell (RBC) membrane components, or against membrane components (autoantibodies) (Figure 1). ${ }^{3,4,6}$ Additionally, some drugs can cause serum proteins, including immunoglobulins, to be adsorbed onto RBC membranes, potentially mediating their destruction. ${ }^{6}$ To date, the best-understood mechanisms of DIIHA involve penicillin and cefotetan, which covalently bind to RBC membranes and induce binding of $\mathrm{IgG}$ antibodies to drug epitopes. These coated RBCs then undergo extravascular destruction via Fcreceptor recognition by splenic macrophages. ${ }^{2}$

A reliable laboratory finding in DIIHA is a positive direct antiglobulin test (DAT; colloquially referred to as a positive direct Coombs' test). ${ }^{2}$ This is a method of detecting the presence of antibodies or complement directly bound to RBC membranes. ${ }^{7}$ However, up to $0.1 \%$ of healthy individuals and up to $15 \%$ of hospitalized patient specimens can have a positive DAT result without evidence of hemolysis

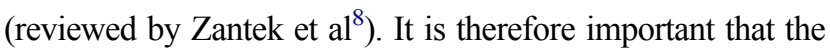
significance of a positive result is interpreted in the context of the clinical situation. ${ }^{8}$

As of 2014, 136 drugs had been reported to cause DIIHA. ${ }^{9}$ Historically, the most common drugs associated with this condition were methyldopa and high-dose intravenous penicillin. ${ }^{10}$ However, over the past 50 years, the spectrum of medications implicated in DIIHA has changed considerably and the focus has shifted to the cephalosporins and other $\beta$-lactam antibiotics, such as piperacillin. ${ }^{9,11}$ Evidence shows that first-generation cephalosporins (eg,

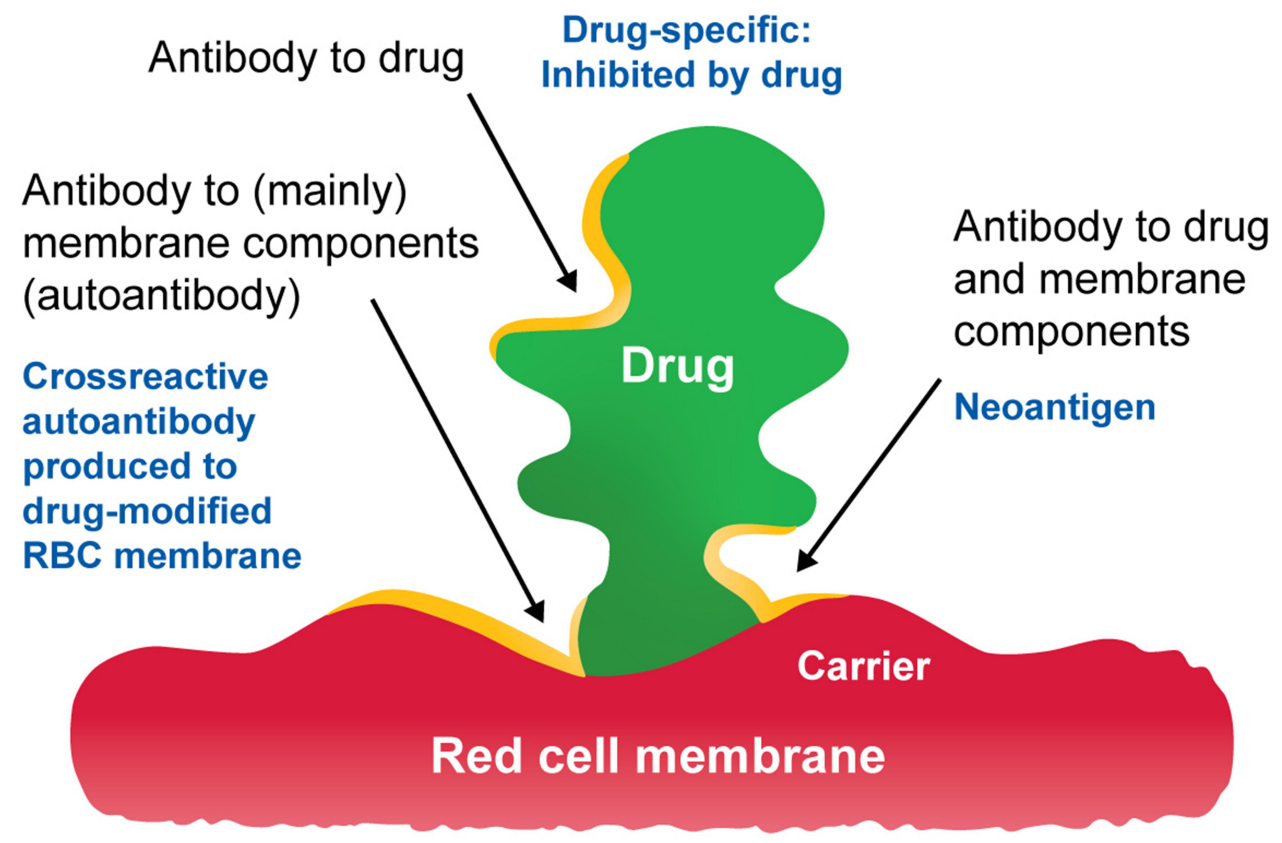

Figure I A proposed unifying model of drug-induced immune hemolytic anemias based on the hapten theory of antibody production. Reproduced with permission from Branch DR. Drug-induced immune haemolytic anaemias. ISBT Sci Ser. 2018;14(I):49-52.@ 2018 International Society of Blood Transfusion. ${ }^{6}$ 
cephalexin, cephalothin, and cefazolin) rarely caused immune hemolytic anemia, with only five welldocumented cases since their first introduction in $1964 .{ }^{12}$ Second- and third-generation cephalosporins, especially cefotetan and ceftriaxone, have been associated with severe, sometimes fatal hemolytic anemia. ${ }^{12}$ In a reference laboratory in the USA, these agents accounted for over $80 \%$ of reported cases of DIIHA over the ten-year period to 2005 , with cefotetan being the most frequently suspected cephalosporin. ${ }^{10}$ Similarly, in a case-control study in Germany, second- and third-generation cephalosporins were a frequent cause of DIIHA. ${ }^{1}$ However, in this study, the proportion of cases caused by cephalosporins was lower than that observed in the USA study. This may reflect the fact that cefotetan was no longer available in Germany during the study period. ${ }^{1}$ Of note, children may be more vulnerable to ceftriaxone-induced hemolytic anemia than adults, and reactions in children can be severe and often fatal. ${ }^{13}$ However, the condition is rare, and affected children commonly have underlying chronic hematologic or immunologic disorders or chronic/recurrent infections (reviewed by Northrop and Agarwal ${ }^{14}$ ). Since 2008, piperacillin has superseded cefotetan and ceftriaxone as the leading cause of DIIHA. ${ }^{9}$

Although hemolytic anemia has not been observed in clinical trials of the advanced-generation cephalosporin, ceftaroline, DAT seroconversion has been reported. ${ }^{15-18}$ In the Phase 3, multicenter, randomized, double-blind FOCUS trials in community-acquired bacterial pneumonia, there were more positive DAT results with ceftaroline versus ceftriaxone $(9.8 \%$ versus $4.5 \%)$, but no difference in the rates of anemia $(0.8 \%$ versus $0.4 \%)$, and no patients in either treatment arm were identified with a clinical presentation of hemolytic anemia. ${ }^{16,17}$ In the Phase 3, multicenter, randomized, double-blind CANVAS trials in acute bacterial skin and skin structure infections (ABSSSIs), 11.6\% of ceftaroline-treated patients had positive DAT results, compared with $4.3 \%$ of patients treated with vancomycin plus aztreonam, with no reports of hemolytic anemia in either group. ${ }^{15}$

Ceftobiprole, the active moiety of the prodrug ceftobiprole medocaril, is an advanced-generation, broad-spectrum cephalosporin, which has a rapid bactericidal effect against both methicillin-sensitive and methicillin-resistant Staphylococcus aureus infections. ${ }^{19}$ It is currently approved in many European and non-European countries for the treatment of community- and hospital-acquired pneumonia (excluding ventilator-associated pneumonia). ${ }^{20}$ DATs were not performed in four previous Phase 3 trials of ceftobiprole; however, there were no adverse events of hemolytic anemia reported in these trials. ${ }^{21-24}$ Also, to date, no cases of hemolytic anemia have been reported in the post-marketing setting, where experience of $>70,000$ patient-days has been recorded (unpublished data).

Here, we review safety data from three recent Phase 3 trials of ceftobiprole (one in pediatric patients with pneumonia, one in adults with ABSSSIs, and one in adults with $S$. aureus bacteremia), with regard to the incidence of positive DAT results and hemolytic anemia.

\section{Patients and Methods \\ Design and Participants}

This analysis includes patients enrolled in three recent or ongoing clinical trials. BPR-PIP-002 (NCT03439124) was a Phase 3, randomized, investigator-blinded, active-controlled, multicenter trial of ceftobiprole $(\mathrm{n}=94)$ versus intravenous standard-of-care cephalosporin \pm vancomycin $(\mathrm{n}=44)$ in patients aged 3 months to $<18$ years with hospital- or communityacquired pneumonia requiring hospitalization and administration of intravenous antibiotics. ${ }^{25}$ TARGET (NCT03137173) was a Phase 3, randomized, double-blind, active-controlled, parallel-group, multicenter, non-inferiority trial of ceftobiprole $(n=335)$ compared with vancomycin plus aztreonam $(n=344)$ in hospitalized patients aged $\geq 18$ years with a diagnosis of ABSSSI, who required intravenous antibacterial treatment. ${ }^{26}$ ERADICATE (NCT03138733) is an ongoing Phase 3, randomized, double-blind, active-controlled, parallel-group, multicenter, trial of ceftobiprole compared with daptomycin \pm aztreonam in hospitalized patients aged $\geq 18$ years with complicated forms of $S$. aureus bacteremia, including infective endocarditis. ${ }^{27}$ As of August 2020, 201 patients have been randomized 1:1 to ceftobiprole or daptomycin \pm aztreonam in this trial. Safety assessments, including adverse event monitoring, physical examination, vital signs, safety laboratory tests, and other assessments, were performed regularly throughout all three trials.

\section{Laboratory Assessments and Methodology}

DATs were performed according to the following schedules: in the BPR-PIP-002 trial, in all children aged $\geq 6$ years and in younger patients not treated with vancomycin, a DAT was performed at screening and at the test of cure (TOC) visit (7-14 days after the end of treatment [EOT]); in TARGET, a DAT was performed at baseline and EOT; and in ERADICATE, 
a DAT was performed at baseline, EOT, and at a posttreatment Day 70 visit.

For the BPR-PIP-002 trial, DATs were performed at Gdańsk Laboratory, Gdańsk, Poland (for samples from patients in Bulgaria, Hungary, and Romania) or at IMD Laboratory, Tbilisi, Georgia (for samples from patients in Georgia). For the TARGET and ERADICATE trials, DATs were performed at PPD Laboratories USA (Kentucky, USA) or PPD Laboratories Europe (Zaventem, Belgium). For all three trials, the DAT performed was an agglutination tube assay using polyspecific antiglobulin sera (BioRad Laboratories, California, USA). For the BPR-PIP-002 trial, positive tests were further analyzed by a semi-quantitative DG Gel card test (Grifols Diagnostics, Barcelona, Spain).

\section{Results}

\section{Direct Antiglobulin Tests BPR-PIP-002}

A total of 102 patients (ceftobiprole $n=72$, ceftriaxone $\mathrm{n}=28$, and ceftazidime $\mathrm{n}=2$ ) had a DAT at baseline and 113 patients had a test at the TOC visit (ceftobiprole $n=74$, ceftriaxone $n=37$, and ceftazidime $n=2$ ) (Table 1 ). One patient had a test at an unscheduled visit on treatment day 9 (ceftobiprole $\mathrm{n}=1$ ).

Of the ceftobiprole patients, a total of 18 recorded a positive DAT result. Nine had a positive result at baseline, five reported seroconversion (negative at baseline and positive at the TOC visit), and a further four had a positive result at the TOC visit but were not tested at baseline (Table 2). Only two patients had an increase in reaction strength from baseline to follow-up visit (the first patient had an increase from $1+$ at baseline to $2+$ at the TOC visit, and the second had an increase from $0.5+$ at baseline to $1+$ at treatment day 9). Of the ceftriaxone patients, a total of two patients recorded a positive DAT result. One had a positive result at baseline, but a subsequent negative result at the TOC visit. The other had a positive result at the TOC visit but was not tested at baseline (Table 2).

\section{TARGET}

In the ceftobiprole group, 289 patients had a DAT at baseline and 291 patients at the EOT visit. In the vancomycin \pm aztreonam group, 280 patients had a test at baseline and 304 patients at the EOT visit. One patient in the vancomycin \pm aztreonam group had a DAT 12 days after EOT. All DATs were negative in both treatment groups (Table 1).
Table I Outcome of Direct Antiglobulin Testing in Phase 3 Clinical Trials of Ceftobiprole

\begin{tabular}{|c|c|c|c|c|c|}
\hline Clinical Trial & \multicolumn{5}{|c|}{$\begin{array}{l}\text { Number of Patients with Positive DAT } \\
\text { Results }(\mathrm{n} / \mathrm{N})\end{array}$} \\
\hline \multirow[t]{2}{*}{ BPR-PIP-002 } & \multicolumn{2}{|c|}{ Ceftobiprole } & \multicolumn{3}{|c|}{$\begin{array}{l}\text { IV SOC cephalosporin }{ }^{\mathrm{a}} \pm \\
\text { vancomycin }\end{array}$} \\
\hline & $\begin{array}{l}\text { Baseline } \\
9 / 72\end{array}$ & $\begin{array}{l}\text { TOC } \\
14 / 75\end{array}$ & $\begin{array}{l}\text { Baseline } \\
1 / 30\end{array}$ & $\begin{array}{l}\mathrm{TO} \\
\mathrm{I} / 3\end{array}$ & \\
\hline \multirow[t]{3}{*}{ TARGET } & \multicolumn{2}{|c|}{ Ceftobiprole } & \multicolumn{3}{|c|}{ Vancomycin \pm aztreonam } \\
\hline & Baseline & EOT & Baseline & EO & \\
\hline & $0 / 289$ & $0 / 291$ & $0 / 280$ & $0 / 3$ & \\
\hline \multirow[t]{3}{*}{ ERADICATE } & \multicolumn{5}{|c|}{$\begin{array}{l}\text { Treatment (ceftobiprole or daptomycin } \pm \\
\text { aztreonam)d }\end{array}$} \\
\hline & Baseline & EOT & \multicolumn{3}{|c|}{$\begin{array}{l}\text { Post-treatment Day } \\
70\end{array}$} \\
\hline & $1 / 187$ & $1 / 163$ & \multicolumn{2}{|l|}{$0 / 127$} & \\
\hline
\end{tabular}

Notes: ${ }^{a}$ Ceftriaxone (baseline: $n=28$, TOC: $n=37$ ) and ceftazidime (baseline: $n=2$, TOC: $\mathrm{n}=2$ ). ${ }^{\mathrm{b}} \mathrm{I}$ patient had a DAT at an unscheduled visit at treatment day $9 .{ }^{\mathrm{C}} \mathrm{I}$ patient had a DAT at an unscheduled visit 12 days after EOT. ${ }^{\mathrm{d}}$ Results cannot be allocated by treatment, as the trial is ongoing and blinded.

Abbreviations: DAT, direct antiglobulin test; $n$, number of patients with positive DAT results; N, number of DATs performed; IV, intravenous; SOC, standard of care; TOC, test of cure; EOT, end of treatment.

\section{ERADICATE}

As the trial is currently ongoing and blinded, the results cannot be reported by treatment group. One patient had a positive DAT result at baseline and at EOT. This patient did not have a test after EOT, including at the Day 70 visit. There was no evidence of hemolysis or hemolytic anemia in this patient at any time during the trial. All other DAT results were negative, including 186 patients at baseline, 162 patients at EOT visit, and 127 patients at the Day 70 visit. A total of 120 patients had negative DAT results both at the EOT and the Day 70 visits, and 114 patients had negative DAT results at all three visits. The results are summarized in Table 1.

\section{Other Laboratory Tests}

For all three trials, results of other blood tests, ie, hemoglobin, hematocrit, reticulocyte count and percent, haptoglobin, lactate dehydrogenase, bilirubin, and hepatic transaminases (alanine aminotransferase, aspartate aminotransferase) showed no evidence of hemolysis or hemolytic anemia in any patient.

\section{Adverse Events}

The adverse event profile for all three trials was consistent with that reported previously for ceftobiprole. ${ }^{21-24}$ No 
Table 2 Breakdown of Positive Direct Antiglobulin Test Results in BPR-PIP-002 Trial by Visit

\begin{tabular}{|l|l|l|l|}
\hline \multicolumn{2}{|l|}{ DAT Results at Baseline and TOC } & $\begin{array}{l}\text { Ceftobiprole } \\
\text { Number of Patients with Tests Performed: } \\
\text { N=72 at Baseline; N=75 at TOC Visit }{ }^{\mathrm{a}}\end{array}$ & $\begin{array}{l}\text { IV SOC Cephalosporin } \pm \text { Vancomycin } \\
\text { Number of Patients with Tests Performed: } \\
\text { Ceftriaxone: 28 at Baseline; 37 at TOC } \\
\text { Ceftazidime: 2 at Baseline; 2 at TOC }\end{array}$ \\
\hline Baseline & TOC $^{\mathrm{a}}$ & \multicolumn{2}{|c|}{ Number of Patients with Positive DAT Results } \\
\hline $\begin{array}{l}\text { Negative } \\
\text { Not performed }\end{array}$ & $\begin{array}{l}\text { Positive } \\
\text { Positive } \\
\begin{array}{l}\text { Positive } \\
\text { Positive }\end{array}\end{array}$ & 5 & 0 \\
\hline
\end{tabular}

Notes: ${ }^{a}$ I patient had a DAT at an unscheduled visit at treatment day $9 .{ }^{b}$ Both patients with a positive DAT result in the SOC cephalosporin treatment group received ceftriaxone.

Abbreviations: DAT, direct antiglobulin test; TOC, test of cure; N, number of tests performed; IV, intravenous; SOC, standard of care.

adverse events of hemolytic anemia were reported in any of the three trials included in this analysis.

\section{Discussion}

In this analysis of three large Phase 3 trials $(n=1018)$, no evidence of hemolytic anemia associated with ceftobiprole was observed in either adults or children across several indications. Furthermore, no patients discontinued ceftobiprole treatment as a result of anemia. Evidence of DAT seroconversion was reported in five children treated with ceftobiprole in the BPR-PIP-002 trial. An additional four children had a positive result at the TOC visit, but a DAT was not conducted at baseline. For each of these cases, there were no other laboratory or clinical findings of hemolytic anemia. No evidence of DIIHA was reported in either the TARGET or ERADICATE trials, echoing results observed with another advancedgeneration cephalosporin, ceftaroline, which reported no cases of DIIHA in several large Phase 3 trials. ${ }^{15-17}$

The differences in the frequency of positive DAT results between the BPR-PIP-002 trial and the TARGET/ ERADICATE trials may reflect the use of different testing laboratories. Alternatively, as the patients were randomized 2:1 (ceftobiprole to standard-of-care cephalosporin) in the BPR-PIP-002 trial, these results may be due to the fact that a larger proportion of ceftobiprole-treated patients were included in this trial compared with TARGET and ERADICATE. It should also be noted that in the BPR-PIP -002 trial, after a minimum of 3 days' treatment with intravenous antibiotics, patients who met standardized criteria for clinical improvement could be switched to an oral antibiotic (penicillins, cephalosporins, macrolides, clindamycin, or linezolid) at the discretion of the blinded investigator, to complete a minimum of 7 days' antibiotic treatment. As such, there may be an influence of the oral antibiotics on the DAT result at the TOC visit. In the TARGET and ERADICATE trials, patients received intravenous antibiotics throughout the treatment course.

In a small number of hospitalized patients described in this analysis (10 in the BPR-PIP-002 trial and 1 in the ERADICATE trial), a positive DAT result was reported at baseline, ie, prior to initiation of treatment. This accounts for $1.3 \%$ of all baseline tests and is consistent with previous reports demonstrating that positive DAT results without evidence of hemolysis can occur in hospitalized patients. ${ }^{8}$ There are a number of potential causes of such results, of varying clinical significance. ${ }^{28}$ These can include older age, malignancy, and some infectious diseases including human immunodeficiency virus infection. ${ }^{7,28}$

There are a number of limitations associated with our analysis. Firstly, drug-dependent antibodies were not measured in patients with a positive DAT in the three trials described here. This may be relevant, as drug-dependent antibodies have previously been detected in patients without clinical evidence of significant hemolytic anemia, eg, in a single-center study, antibodies to piperacillin were detected in two out of 43 (4.7\%) patients with cystic fibrosis; of these, one patient developed a mild immune hemolytic anemia. ${ }^{29}$ Secondly, in the BPR-PIP-002 trial, the DAT was performed 7-14 days after the end of treatment and therefore ceftobiprole would not be present in the sample. Of note, this should not impact the results from the TARGET and ERADICATE trials, where the DAT was performed at EOT.

\section{Conclusions}

Consistent with previous Phase 3 trials, the evidence presented here indicates that ceftobiprole administration may not 
be associated with DIIHA. A small number of positive DAT results were reported without evidence of hemolysis; this is consistent with previous reports from hospitalized patients.

\section{Data Sharing Statement}

All data relevant to the analysis are included in the article. The deidentified datasets used and analyzed during the current analysis are available from the corresponding author at any time upon reasonable request.

\section{Ethics Approval and Informed Consent}

All three trials described in this manuscript were conducted in compliance with applicable laws and regulations, ICH Good Clinical Practice guidelines and the Declaration of Helsinki, and the protocols were approved by the relevant Independent Ethics Committees/Institutional Review Boards (Supplementary Table 1). For adult patients, written informed consent was required prior to the conduct of any trial procedures. For pediatric patients, each child's parent or legal guardian provided written informed consent. If appropriate, the child's assent was also sought prior to participation in the trial.

\section{Acknowledgments}

The authors are grateful to the investigators, site personnel, patients, and parents who participated in these trials. Under the direction of the authors, medical writing support for the manuscript was provided by Stephanie Carter of Arc, a division of Spirit Medical Communications Group Limited, funded by Basilea Pharmaceutica International Ltd.

\section{Author Contributions}

$\mathrm{KH}$ contributed to analysis conceptions and designs, and acquisition of data. All authors made substantial contributions to analysis of data, took part in drafting the article or revising it critically for important intellectual content, agreed to submit to the current journal, gave final approval of the version to be published, and agreed to be accountable for all aspects of the work.

\section{Disclosure}

$\mathrm{KH}, \mathrm{TW}$, and MAG are employees of Basilea Pharmaceutica International Ltd. The authors report no other conflicts of interest in this work.

\section{References}

1. Garbe E, Andersohn F, Bronder E, et al. Drug induced immune haemolytic anaemia in the Berlin Case-Control Surveillance Study. Br J Haematol. 2011;154(5):644-653. doi:10.1111/j.1365-2141.2011.08784.x

2. Pierce A, Nester T. Pathology consultation on drug-induced hemolytic anemia. Am J Clin Pathol. 2011;136(1):7-12. doi:10.1309/ AJCPBVLJZH6W6RQM

3. Habibi B. Drug induced red blood cell autoantibodies co-developed with drug specific antibodies causing haemolytic anaemias. Br J Haematol. 1985;61(1):139-143. doi:10.1111/j.1365-2141.1985.tb04069.x

4. Mueller-Eckhardt C, Salama A. Drug-induced immune cytopenias: a unifying pathogenetic concept with special emphasis on the role of drug metabolites. Transfus Med Rev. 1990;4(1):69-77. doi:10.1016/ S0887-7963(90)70249-0

5. Salama A. Drug-induced immune hemolytic anemia. Expert Opin Drug Saf. 2009;8(1):73-79. doi:10.1517/14740330802577351

6. Branch DR. Drug-induced immune haemolytic anaemias. ISBT Sci Ser. 2019;14(1):49-52. doi:10.1111/voxs. 12469

7. Parker V, Tormey CA. The direct antiglobulin test: indications, interpretation, and pitfalls. Arch Pathol Lab Med. 2017;141(2):305-310. doi:10.5858/arpa.2015-0444-RS

8. Zantek ND, Koepsell SA, Tharp DR Jr, Cohn CS. The direct antiglobulin test: a critical step in the evaluation of hemolysis. $\mathrm{Am}$ J Hematol. 2012;87(7):707-709. doi:10.1002/ajh.23218

9. Garratty G, Arndt PA. Drugs that have been shown to cause drug-induced immune hemolytic anemia or positive direct antiglobulin tests: some interesting findings since 2007. Immunohematology. 2014;30(2):66-79.

10. Arndt PA, Garratty G. The changing spectrum of drug-induced immune hemolytic anemia. Semin Hematol. 2005;42(3):137-144. doi:10.1053/j.seminhematol.2005.04.004

11. Mayer B, Bartolmäs T, Yürek S, Salama A. Variability of findings in drug-induced immune haemolytic anaemia: experience over 20 years in a single centre. Transfus Med Hemother. 2015;42(5):333-339. doi: $10.1159 / 000440673$

12. Arndt PA, Leger RM, Garratty G. Serology of antibodies to second- and third-generation cephalosporins associated with immune hemolytic anemia and/or positive direct antiglobulin tests. Transfusion. 1999;39(11-12):1239-1246. doi:10.1046/j.1537-2995.1999.39111239.x

13. Arndt PA, Leger RM, Garratty G. Serologic characteristics of ceftriaxone antibodies in 25 patients with drug-induced immune hemolytic anemia. Transfusion. 2012;52(3):602-612. doi:10.1111/ j.1537-2995.2011.03321.x

14. Northrop MS, Agarwal HS. Ceftriaxone-induced hemolytic anemia: case report and review of literature. J Pediatr Hematol Oncol. 2015;37(1):e63-66. doi:10.1097/MPH.0000000000000181

15. Corrado ML. Integrated safety summary of CANVAS 1 and 2 trials: phase III, randomized, double-blind studies evaluating ceftaroline fosamil for the treatment of patients with complicated skin and skin structure infections. J Antimicrob Chemother. 2010;65(Suppl 4): iv67-iv71. doi:10.1093/jac/dkq256

16. Goodman JJ, Martin SI. Critical appraisal of ceftaroline in the management of community-acquired bacterial pneumonia and skin infections. Ther Clin Risk Manag. 2012;8:149-156.

17. Rank DR, Friedland HD, Laudano JB. Integrated safety summary of FOCUS 1 and FOCUS 2 trials: phase III randomized, double-blind studies evaluating ceftaroline fosamil for the treatment of patients with community-acquired pneumonia. J Antimicrob Chemother. 2011;66(Suppl 3):iii53-iii59. doi:10.1093/jac/dkr099

18. Allergan. TEFLARO ${ }^{\circledR}$ (ceftaroline fosamil) prescribing information. 2019. Available from: https://media.allergan.com/actavis/actavis/ media/allergan-pdf-documents/product-prescribing/Teflaro-USPI-09_ 2019-2.pdf. Accessed August, 2020. 
19. Liapikou A, Cilloniz C, Torres A. Ceftobiprole for the treatment of pneumonia: a European perspective. Drug Des Devel Ther. 2015;9:4565-4572.

20. Electronic Medicines Compendium. Zevtera $500 \mathrm{mg}$ powder for concentrate for solution for infusion. 2018. Available from: https://www.medicines.org.uk/emc/product/9164. Accessed August, 2020.

21. Noel GJ, Strauss RS, Amsler K, Heep M, Pypstra R, Solomkin JS. Results of a double-blind, randomized trial of ceftobiprole treatment of complicated skin and skin structure infections caused by gram-positive bacteria. Antimicrob Agents Chemother. 2008;52 (1):37-44. doi:10.1128/AAC.00551-07

22. Noel GJ, Bush K, Bagchi P, Ianus J, Strauss RS. A randomized, double-blind trial comparing ceftobiprole medocaril with vancomycin plus ceftazidime for the treatment of patients with complicated skin and skin-structure infections. Clin Infect Dis. 2008;46(5):647-655. doi:10.1086/526527

23. Nicholson SC, Welte T, File TM Jr, et al. A randomised, double-blind trial comparing ceftobiprole medocaril with ceftriaxone with or without linezolid for the treatment of patients with community-acquired pneumonia requiring hospitalisation. Int $J$ Antimicrob Agents. 2012;39(3):240-246. doi:10.1016/j.ijantimicag.2011.11.005

24. Awad SS, Rodriguez AH, Chuang YC, et al. A phase 3 randomized double-blind comparison of ceftobiprole medocaril versus ceftazidime plus linezolid for the treatment of hospital-acquired pneumonia. Clin Infect Dis. 2014;59(1):51-61. doi:10.1093/cid/ciu219
25. NCT03439124. Ceftobiprole in the treatment of pediatric patients with pneumonia. 2020. Available from: https://clinicaltrials.gov/ct2/ show/NCT03439124. Accessed August, 2020.

26. Overcash J, Kim C, Keech R, et al. Ceftobiprole compared with vancomycin plus aztreonam in the treatment of acute bacterial skin and skin structure infections: results of a Phase 3, randomized, double-blind trial (TARGET). Clin Infect Dis. In press 2020. doi:10.1093/cid/ciaa974

27. Hamed K, Engelhardt M, Jones ME, et al. Ceftobiprole versus daptomycin in Staphylococcus aureus bacteremia: a novel protocol for a double-blind, phase III trial. Future Microbiol. 2020;15:35-48. doi:10.2217/fmb-2019-0332

28. Kim TO, Grimes AB, Kirk S, et al. Association of a positive direct antiglobulin test with chronic immune thrombocytopenia and use of second line therapies in children: a multi-institutional review. Am J Hematol. 2019;94(4):461-466. doi:10.1002/ajh.25409

29. Roehmel J, Specht P, Staab D, Schwarz C, Salama A, Mayer B. Risk of piperacillin-induced hemolytic anemia in patients with cystic fibrosis and antipseudomonal treatment: a prospective observational study. Transfusion. 2019;59(12):3746-3754. doi:10.1111/trf.15591
Infection and Drug Resistance

\section{Publish your work in this journal}

Infection and Drug Resistance is an international, peer-reviewed openaccess journal that focuses on the optimal treatment of infection (bacterial, fungal and viral) and the development and institution of preventive strategies to minimize the development and spread of resistance. The journal is specifically concerned with the epidemiology of
Dovepress

antibiotic resistance and the mechanisms of resistance development and diffusion in both hospitals and the community. The manuscript management system is completely online and includes a very quick and fair peerreview system, which is all easy to use. Visit http://www.dovepress.com/ testimonials.php to read real quotes from published authors. 